\title{
Primitive Gastric Lymphoma - a Series of 3 Cases
}

\author{
DRAGOS VOICU ${ }^{1}$, CONSTANTIN VOLOVAT ${ }^{2 *}$, SIMONA RUXANDRA VOLOVAT $^{2 *}$, ADRIAN BEZNEA ${ }^{1}$, \\ BIANCA CONSTANTIN ${ }^{3 *}$, LAURA REBEGEA ${ }^{4,5}$, DOREL FIRESCU ${ }^{1,6}$, BOGDAN ALEXANDRU CIUBARA ${ }^{1}$, \\ RAUL MIHAILOV ${ }^{1}$, DAN CONSTANTINESCU ${ }^{2}$, CRISTINA SERBAN ${ }^{1,6}$ \\ ${ }^{1}$ Dunarea de Jos University of Galati, Faculty of Medicine and Pharmacy, Surgical Clinical Department, 47 Domneasca Str., \\ 800008, Galati, Romania \\ ${ }^{2}$ Grigore T. Popa University of Medicine and Pharmacy, III-rd Medical Department, 16 Universitatii Str., 700115, Iaşi, \\ Romania \\ ${ }^{3}$ Dunarea de Jos University of Galati, Faculty of Medicine and Pharmacy, Morphological and Functional Sciences \\ Department, 47 Domneasca Str., 800008, Galati,Romania \\ ${ }^{4}$ Sf. Ap. Andrei Emergency Clinical Hospital, Department of Radiotherapy, 177 Brailei Str., 800578, Galati, Romania \\ ${ }^{5}$ Dunarea de Jos University of Galati, Faculty of Medicine and Pharmacy, Medical Clinical Department, 47 Domneasca Str., \\ 800008, Galati, Romania \\ ${ }^{6}$ Sf. Ap. Andrei Emergency Clinical Hospital, Surgery Clinic II,177 Brailei Str., 800578, Galati, Romania
}

The Primitive Gastric Lymphoma represents the extra-ganglionic non-Hodgkin lymphoma most frequently and comprises a large spectrum, ranging from the mucosa-associated lymphoid tissue (MALT) lymphoma to aggressive diffuse large B-cell lymphoma. The Primitive Gastric Lymphoma is a rare tumor, with an occurrence ranging from $4 \%$ to $20 \%$ of non-Hodgkin lymphomas and approximatively $5 \%$ of primary gastric neoplasms. The chronic gastritis secondary to the infection with Helicobacter pylori was considered a major predisposing factor for MALT lymphoma. The clinical evolution and the prognosis of this disease depend on the subtype and histopathological stage at the moment of the diagnosis. Due to its rarity, many aspects of this neoplasm remain still controversial.

Keywords: lymphoma, primitive, Helicobacter pylori

MALT lymphomas are neoplasms which develop from the lymphoid tissue associated to the visceral mucosa (pharynx, gastro-intestinal duct, rectum, urinary bladder). The Primitive Gastric Lymphoma is a rare tumor (5\% of the total of gastric neoplasms), but it represents at the same time the most frequent extra-ganglionic lymphoma (4-20\%) [1,2]. The etiology of the disease is still unclear, but the involvement of the infection with Helicobacter pylori has become a certainty. The action mechanism of Helicobacter pylori is indirect: it initially provokes the occurrence of gastritis, subsequently followed by the aggregation of T CD4 lymphocytes + and of B lymphocytes in own gastric lamina [3-5].

The gastric lymphoma develops after 50 years old (average 60 - 62 years old), mostly at men (2:1) [6].

Discovered usually too late due to rough symptomatology, the role of first intent surgery in the treatment of the Primitive Gastric Lymphoma is absolute, while for the cases diagnosed in incipient phases, the oncological treatment (chemotherapy + radiotherapy) can obtain similar results in what concerns the survival.

\section{Experimental part}

Case reports

Case no. 1

Male patient of 62 years old, smoker and constant moderate user of ethanol, is admitted to hospital due to presenting anorexia, dyspepsia and weight loss (around $10 \mathrm{~kg}$ ) for approximately two months. From his medical history, we mention appendectomy performed at 12 years old and infection with HBV detected 15 years ago. The clinical examination noted only a sensibility at deep palpation of the epigastrium. Upper Digestive Endoscopy (UDE) showed the rigidity and alteration of the relief of small gastric curvature on both sides, with friable mucosa, presenting hemorrhagic exulcerations. CT scan observed the thickening of the wall along the gastric antrum with the retraction of the small curvature and lymphadenomegaly along the same line.

When opening the peritoneal cavity, it was observed the presence of a voluminous tumor mass, of increased thickness, which involves the small antral curvature, but infiltrates also the vertical wall, up to $5 \mathrm{~cm}$ from the esogastric junction and perigastricadenopathy, without splenomegaly. Total gastrectomy was performed, restoring the digestive continuity by esojejunal terminal - lateral anastomosis on the loop in Y.

*email: constantin.volovat@umfiasi.ro; simona.volovat@umfiasi.ro; constantin_bianca2009@yahoo.com 
Histopathologic examination: non-Hodgkin B cell-lymphoma, stage IIE1, with high degree of malignity, associated with antral atrophic chronic gastritis and complete multifocal intestinal metaplasia, with infection of H.pylori. 4 out of those 22 lymphatic ganglions removed at the same time with the perivisceral adipose tissue were affected by neoplasia. The resection margins were not affected.

The evolution after surgery was favorable, with discharge from hospital after 14 days from the surgery. Taken over by Oncology, where he underwent adjuvant chemotherapy, the patient manages to maintain his weight at an acceptable level, without signs of relapse after 18 months.

\section{Case no. 2}

Female patient of 82 years old with posterior - inferior Acute Myocardial Infarction and IVS II NYHA in her medical history, overweight, on anticoagulant chronic treatment, is admitted to hospital for repeated lypotimic episodes, followed by melenic stools. 5 years ago, on the occasion of a hospitalization in Cardiology Ward, she had performed an endoscopic examination, by which it was observed the presence of a gastric ulceration of small curvature, a light reflux esophagitis and infection associated with Helicobacter Pylori. The clinical examination noted a sensibility at the profound palpation of the epigastrium and right hypocondrium and confirmed the melena. The laboratory examination highlighted the anemia ( $\mathrm{Hb}$ 8.6-10.2 $\mathrm{g} \mathrm{\% )}$ and INR 1.45. UDE found the congestive alteration of mucous surface of the small curvature, on both sides, starting from $10 \mathrm{~cm}$ subcardial, up to pylorus; at gastric angle level, ulceration $(1 \mathrm{~cm}$ diameter $)$ with irregular margins. Multiple biopsies were performed and the histological examination of the mucous fragments had diagnosed a non-Hodgkin lymphoma of the stomach, derived from peripheral B lymphocytes, with a low degree of malignity. The CT scan confirmed the presence of an infiltrative tumor mass, with lymphadenomegaly along the small gastric curvature and a small hypo-density of cystic aspect in the upper tierce of the spleen.

When opening the peritoneal cavity, it was observed a gastric wall of the small curvature thickened at antral level and on the vertical portion up to about $10 \mathrm{~cm}$ from the eso-gastric junction, with perigastricadenopathy at this level. The biopsy of the lesion, followed by an anatomic - pathologic extemporaneous examination, confirmed the implication of the gastric wall in a lympho-proliferative process. Taking into consideration the age of the patient and the associated flaws, subtotal gastrectomy was chosen, with the removal of the tumor and ganglionic masses and restoration of the digestive continuity by Hoffmeister-Finsterer gastrojejunal anastomosis.

Final histopathologic diagnosis: ulcerated non-Hodgkin lymphoma, with peripheral cells of B type, stage IIE2, extended in all wall thickness, including serous, lympho-ganglions of the small curvature and perigastric adipose tissue. The proximal resection margin was affected.

The immediate evolution after surgery was favorable, with discharge after 18 days from the surgery. After 3 more weeks from leaving the hospital, while at home, the patient underwent extended Myocardial Infarction, with fast decompensation and irresuscitable cardiac arrest, followed by exitus in Emergency Admission Unit (EAU), where she arrived by Ambulance.

\section{Case no. 3}

A 46 years old male patient, a smoker and a constant ethanol consumer, is admitted to hospital, having for about 6 months anorexia and an upper abdominal rough dyspeptic syndrome followed by a weight loss (about $8 \mathrm{~kg}$ ). From his medical history, it is noted the appendectomy performed in adolescence and a liver steatosis, diagnosed and treated in the outpatient clinic by the Family Doctor. At the clinical examination, he presented sensitivity at deep palpation of the epigastrium. UDE has found rigidity and alteration of the relief of the small gastric curvature, on both sides, with the fleshy mucosa, presenting multiple hemorrhagic exulcerations. The microscopic examination of sampled biopsies only specified the existence of gastric neoplasia, probably adenocarcinoma. CT scan found the tumor thickening with the retraction of the small curvature and lymphadenomegaly along it.

At the opening of the peritoneal cavity, it is observed an infiltrative tumor mass of increased thickness, involving the small antral curvature, with an extension of about $10 \mathrm{~cm}$ above the angle. Subtotal gastrectomy was performed with restoration of digestive continuity through terminal - lateral gastrojejunal anastomosis on the loop in Y.

Histopathological examination: non-Hodgkin B cell-lymphoma, Stage IIE1, with low grade of malignancy, associated with antral atrophic chronic gastritis with $\mathrm{H}$. pylori.

The evolution after surgery was favorable, with discharge after 10 days after surgery. Taken over by Oncology, where he underwent adjuvant chemotherapy, the patient remains eutrophic, without digestive complaints or signs of relapse after 10 months. 


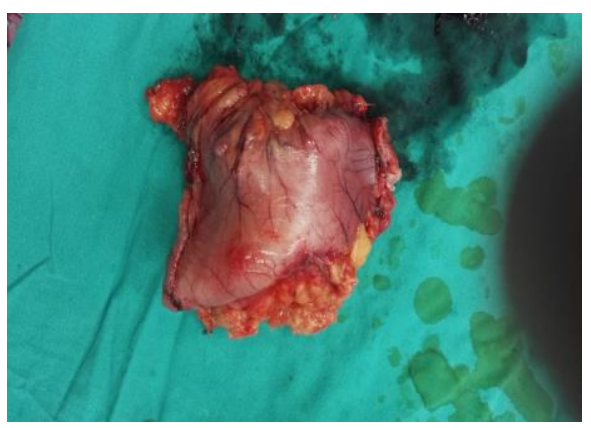

Fig 1. Gastric tumor of small curvature (Primitive Gastric Lymphoma) - surgical snecimen

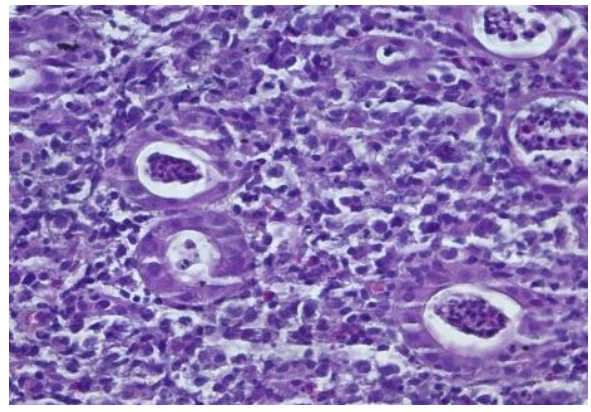

Fig 4. Non-Hodgkin Gastric Lymphoma, with large B cells (obx40, HE)

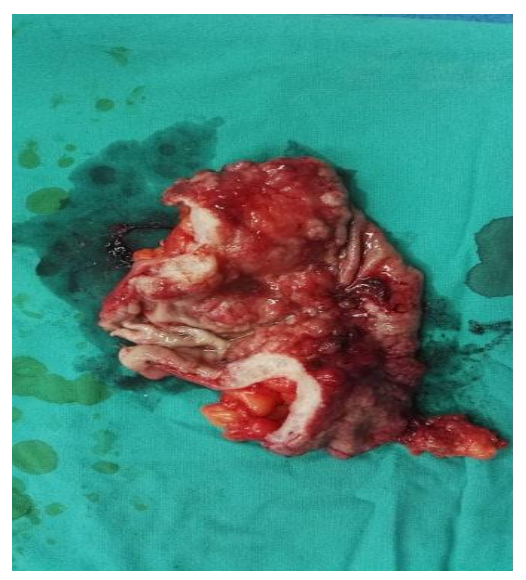

Fig 2. Primary Gastric Lymphoma surgical specimen (endolumenal aspect)

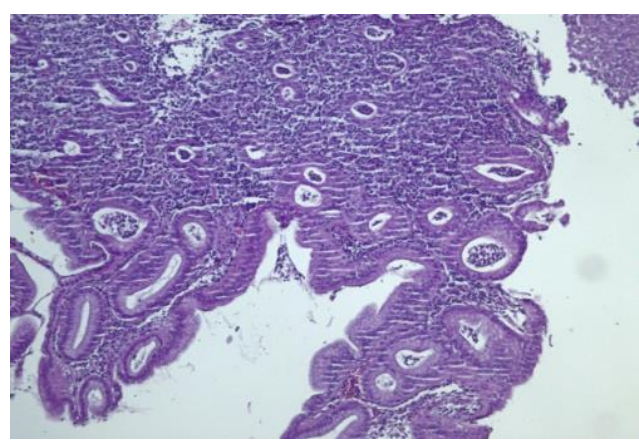

Fig 3. Non-Hodgkin Gastric Lymphoma, with large B cells (obx20, HE)

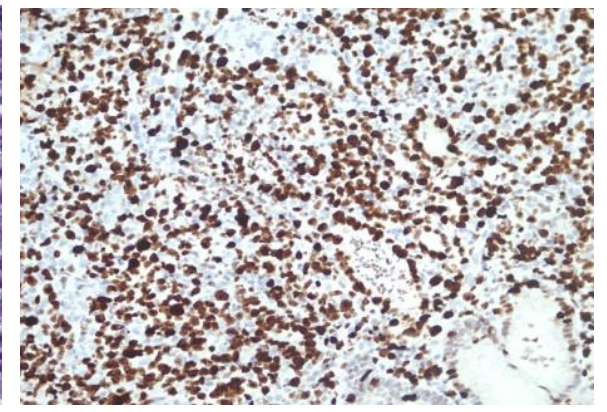

Fig 5 Non-Hodgkin Gastric Lymphoma with large B cells, Ki67-70\%, (obx20 coloration IHC for Ki67)

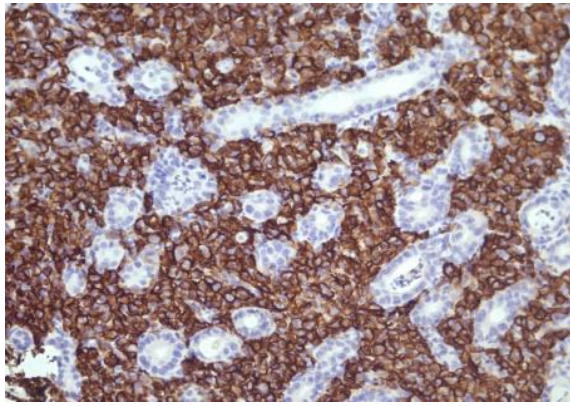

Fig 6. Non-Hodgkin Gastric Lymphoma with large B cells CD20 positively diffused (obx20, coloration IHC for CD20)

\section{Results and discussions}

MALT lymphomas are positive for surface immunoglobulins (Ig), for pan-B antigens (CD19, CD20 and CD79a) and for typical marginal antigen (CD35 and CD21) [7].

CD20 is the membrane marker of mature B lymphocytes. In our cases, we found that the tumor cells diffusely and intensely delineated this marker.

Ki-67 is a non-histone protein identified for the first time in 1991 by Gerdes et al. Because it is expressed throughout the cell cycle phase, except for the resting phase $(\mathrm{G} 0)$, it has been used as a proliferation marker in many cancers including lymphoma [8].

The multimodal therapy combining the surgical resection of the tumor with the chemotherapy and, occasionally, with radiotherapy, is accepted as the primary method of treatment.

Although there are authors taking into consideration the conservative treatment exclusively (chemotherapy, radiotherapy, antibiotherapy to eradicate H. Pylori infection) in Gastric Primitive Lymphoma in the early stages of the disease, it seems that if it is set up early, it is only a way to facilitate the surgery, the only one that can effectively control the disease. In advanced stages, IIIE-IVE, chemotherapy is the treatment of choice, as a first intention, whether or not associated with radiotherapy, as the extension and aggressiveness of the tumor can no longer be surgically controlled $[9,10]$.

Chlorambucil, cyclophosphamide, fludarabine or CHOP association is used. Alfa-2a interferon is very useful in conjunctival localization and rituximab or anti-CD20 monoclonal antibodies have proved to be useful in inducing remission in patients with MALT lymphoma which did not respond to the therapy with antibiotics [11].

MALT Primitive Gastric Lymphoma has a good prognosis when it is diagnosed before the age of 65, having a low degree of malignancy and the surgical resection respects oncological safety margins, with total tumor removal, as compared to those over 65 years old, when the prognosis is low and they are admitted in the performative stage of the 
tumor [12-15]. Survival rates to 5 years are over $90 \%$, if the malignancy is low and over $60 \%$ if the degree of malignancy is high [16].

\section{Conclusions}

The endobioptic gastric exploration, complemented by CT scan, gives an accurate diagnosis in Gastric Lymphoma. The etiological role of chronic gastric infection with $\mathrm{H}$. pylori is certain.

Multimodal treatment with surgery as a first-choice option is almost universally accepted for the initial stages of the disease.

Exclusive conservative treatment (chemotherapy + radiotherapy) addresses to advanced stages.

The prognosis of Primitive Gastric Lymphoma is better than that of adenocarcinoma.

\section{References}

1.D'AMORE, F., CHRISTENSEN, B.E., THORLING, K., PEDERSEN, M., JENSEN, M.K., BOESEN, A.M., ANDERSEN, E., JOHANSEN, P., MORTENSEN, L.S., Incidence, presenting features and prognosis of low-grade B-cell non-Hodgkin's lymphomas. Population-based data from a Danish lymphoma registry, Leuk Lymphoma, 1993, 12(1-2):69-77.

2.REBEGEA, L.F., PATRASCU, A., MIRON, D., DUMITRU, M.E., FIRESCU, D., Metachronous gastrointestinal stromal tumor associated with other neoplasia - case presentation, Romanian Journal of Morphology \& Embryology, 2016;57(4):1429-1435, ISSN(print): 1220-0522, ISSN(online): 2066-8279

3.FERRERI, A.J., ZUCCA, E., Marginal-zone lymphoma, CritRevOncolHematol, 2007, 63(3):245-256

4.NAKAMURA, S., SUGIYAMA, T., MATSUMOTO, T., et al.; JAPAN GAST Study Group. Long-term clinical outcome of gastric MALT lymphoma after eradication of Helicobacter pylori: a multicenter cohort follow-up study of 420 patients in Japan. Gut. 2012; 61(4):507-513.

5.ZULlO, A., HASSAN, C., RIDOLA, L., REPICI, A., MANTA, R., ANDRIANI, A., Gastric MALT lymphoma: old and new insights. Ann Gastroenterol. 2014; 27(1):27-33.

6.GURNEY KA, CARTWRIGHT RA, GILMAN EA, Descriptive epidemiology of gastrointestinal non-Hodgkin's lymphoma in a population-based registry, Br J Cancer, 1999, 79(11-12):1929-1934.

7.PONZONI, M, FERRERI, AJ, PRUNERI, G, et al. Prognostic value of bcl-6, CD10 and CD38 immunoreactivity in stage I-II gastric lymphomas: identification of a subset of CD10+ large B-cell lymphomas with a favorable outcome. Int J Cancer. 2003; 106(2):288-291.

8.GERDES, J., LI, L., SCHLUETER, C., et al. Immunobiochemical and molecular biologic characterization of the cell proliferation-associated nuclear antigen that is defined by monoclonal antibody Ki-67.Am J Pathol 1991;138:867-73

9.AL-AKWAA, A.M., SIDDIQUI, N., AL-MOFLEH, I.A., Primary gastric lymphoma. World J Gastroenterol. 2004; 10(1):5-11. [PMID: 14695759].

10. KOCH, P., Treatment results in localized primary gastric lymphoma: data of patients registered within the German multicenter study (GIT NHL 02/96). JCO. 2005; 23(28):7050-7059

11. GHIMIRE, P., WU, G.Y., ZHU, L., Primary gastrointestinal lymphoma. World J Gastroenterol. 2011; 17(6):697-707.

12. AVILES, A., NAMBO, M.J., NERI, N., et al. The role of surgery in primary gastric lymphoma: results of a controlled clinical trial. Ann Surg. 2004;240(1):44-50

13. TRUS, C., MUNTEANU, M., DIACONU, G., BEZNEA, A., POP, A., Venous thrombectomy - treatment of entero-mesenteric near total venous acut infarct CHIRURGIA 2010; 105 (2): 415-418

14. BEZNEA, A., TRUS, C.T., CHICOS, S.C., CHEBAC, G.R., CEAUSU, M., Peritoneal malignant mesothelioma. CHIRURGIA 2009; 104(2):227230.

15. FIRESCU, D., SERBAN, C., NECHITA, A., DUMITRU, M., REBEGEA, L., Age influence in the prognosis of bacterial secondary peritonitis, Rev.Chim.(Bucharest), 68, no.5, 2017, p.1023-1027

16. THIEBLEMONT, C., CASCIONE, L., CONCONI, A., et al. A MALT lymphomaprognosticindex. Blood. 2017; 130(12):1409-1417. [PMID: 28720586].

Manuscript received: 10.01 .2020 